\title{
Probabilidad de reforzamiento diferencial y no diferencial en una tarea de discriminación condicional ${ }^{*}$
}

\section{Differential and Non-Differential Probability of Reinforcement in a Conditional Discrimination Task}

\author{
CARlos Javier Flores-Aguirre ${ }^{* *}$ \\ REBECA MATEOS-MORFÍN \\ Universidad de Guadalajara, Centro de Estudios \\ e Investigaciones en Comportamiento, México
}

Para citar este artículo. Flores-Aguirre, C.J.\& Mateos-Morfín, R. (2010). Probabilidad de reforzamiento diferencial y no diferencial en una tarea de discriminación condicional. Universitas Psychologica, 9 (2), 485-493.

* Este trabajo fue posible gracias al financiamiento recibido por parte del Programa de Mejoramiento del Profesorado (PROMEP) al proyecto de investigación PROMEP-PTC-336, y al apoyo otorgado al proyecto 104902 del CONACYT.

** La correspondencia relacionada con este trabajo puede enviarse a cualquiera de los autores a Centro de Estudios e Investigaciones en Comportamiento, Francisco de Quevedo No. 180, Col. Arcos Vallarta, Guadalajara, Jalisco, México, 44130. Correo electrónnico: carlos.flores@cucba.udg.mx orebecamateos@gmail.com

\section{RESUMEN}

Empleando una tarea de discriminación condicional, para un grupo de ratas (CD), se reforzaron las respuestas correctas con diferentes probabilidades de reforzamiento ( 0.8 vs. 0.4 ) dependiendo de la señal condicional con la que iniciaba el ensayo; en otro grupo (CND), se reforzaron con la misma probabilidad (0.6). Después de la adquisición, se introdujo un intervalo de retención y una demora de reforzamiento de 4 s. Los sujetos del grupo CD tuvieron una adquisición más rápida y superiores índices de discriminación que los del grupo CND. La introducción del intervalo de retención resultó en un mayor decremento en el índice de discriminación, en los sujetos del grupo CND; la demora de reforzamiento no mostró diferencias en la precisión en ambos grupos. Se extiende la generalidad del efecto de consecuencias diferenciales, mediante la probabilidad de reforzamiento en una tarea de elección con ratas. Los resultados se discuten atendiendo a la ausencia de efectos por introducir la demora de reforzamiento.

Palabras clave autores

Discriminación condicional, efecto de consecuencias diferenciales, probabilidad de reforzamiento, ratas.

Palabras clave descriptores

Respuesta condicionada, condicionamiento operante, experimentación con animales.

\section{A B S T R A C T}

Using a conditional discrimination task, the correct responses for a group of rats (DO) were reinforced with different probabilities (0.8 versus 0.4 ) depending the conditional cue; whereas for another group (NDO) the correct responses were reinforced with the same probability of reinforcement (0.6). After the training (acquisition), the retention interval and the delay of reinforcement were introduced in different phases. The subjects trained with differential outcomes (DO) had a faster acquisition and reached higher discrimination index than non-differential group (NDO). The retention interval results in lower discrimination index in NDO group than in DO group; no differential changes in accuracy were observed by the delay of reinforcement. The results extend the differential outcomes effect to a twochoice conditional discrimination task with rats varying the probability of reinforcement. The absence of delay of reinforcement effect is discussed. Key words authors

Conditional Discrimination, Differential Outcome Effect, Probability of Reinforcement, Rats.

Key words plus

Conditioned Responses, Operant Conditioning, Animal Experimentation. 
En el campo de la discriminación condicional se ha reportado que el aprendizaje de los sujetos se puede ver potenciado, por el reforzamiento diferencial de las respuestas correctas; este efecto se ha reportado en la literatura como Efecto de Consecuencias Diferenciales (ECD) y hace referencia a una mayor velocidad de adquisición y elevados niveles terminales de discriminación, comparado con sujetos que no reciben un entrenamiento con reforzamiento diferencial (para una revisión detallada del tema véanse, Goeters, Blakely \& Poling, 1992; Urcuioli, 2005).

El ECD se ha reportado empleando una variación extremadamente simple de los procedimientos o tareas comúnmente usadas, para evaluar el aprendizaje de discriminaciones condicionales (Pérez, 2001). En el procedimiento típico o estándar, se refuerza la respuesta que se emite ante uno de dos estímulos dado un tercer estímulo antecedente o concurrente, es decir, se refuerza la respuesta ante uno de dos estímulos de comparación (ECO1) dada la ocurrencia de un estímulo muestra (EM1), reforzando la respuesta ante el otro estímulo de comparación (ECO2) dada la ocurrencia de otro estímulo muestra (EM2). La característica de esta tarea y que representa la diferencia con el procedimiento de consecuencias diferenciales es que las respuestas ante el ECO1 dado el EM1 y ante el ECO2 dado el EM2, tienen la misma consecuencia (Cumming \& Berryman, 1961, 1965). En el procedimiento de consecuencias diferenciales las respuestas al ECO1 dado el EM1 tienen una consecuencia diferente a las respuestas emitidas al ECO2 dado el EM2.

El ECD se ha reportado en la literatura usando diferentes tipos de consecuencias: agua vs. comida (Brodigan \& Peterson, 1976; Peterson, Wheeler $\&$ Armstrong, 1978), reforzamiento demorado vs. inmediato (Carlson \& Wielkiewicz, 1972), diferentes duraciones de intervalos entre ensayos (Flores, Arriaga \& Ortiz, 2006), comida + luz comedero vs. luz comedero (Alling, Nickel \& Poling, 1991; Peterson, Wheeler \& Trapold, 1980), continuidad vs. intermitencia de una descarga eléctrica (Overmier, Bull \& Trapold, 1971), magnitud de reforzamiento (Ortíz \& Flores, 2007) variaciones en la localiza- ción del reforzador (Williams, Butler \& Overmier, 1990), distintas probabilidades de reforzamiento (DeLong \& Wasserman, 1981), entre otras.

Una característica adicional a la mayor velocidad de adquisición cuando se emplean procedimientos con consecuencias diferenciales, es que se presenta un menor decremento en el porcentaje de respuestas correctas cuando se incrementa el intervalo entre estímulos EM-ECO, intervalo de retención o intervalo de elección (e.g., Brodigan \& Peterson, 1976, Chatlosh \& Wasserman, 1992; Overmier, Savage \& Sweeney, 1999; Ramos \& Savage, 2003)

Por ejemplo, DeLong y Wasserman (1981) entrenaron un grupo de sujetos con reforzamiento diferencial (D), las respuestas dada una secuencia entre estímulos (EM1-ECO1), luz de color verdelínea vertical estuvo asociada a una probabilidad de reforzamiento de (0.2), mientras que las respuesta dada una secuencia (EM2-ECO2), luz de color rojo-línea horizontal se asociaron con una probabilidad de reforzamiento de (1.0). Para el grupo con reforzamiento no diferencial (ND), las respuestas ante cualquiera de las dos secuencias de estímulo estaban asociadas a una probabilidad de reforzamiento de (0.6). En diferentes ensayos se introdujeron intervalos de demora de 0,5 o 10 s. Reportaron que la velocidad de adquisición fue mayor para el grupo entrenado con consecuencias diferenciales (D). La introducción de la demora entre estímulos (i.e., intervalo de retención) resultó en decrementos en el índice de discriminación (i.e., porcentaje de respuestas correctas) en ambos grupos; sin embargo, los decrementos fueron sustancialmente más marcados para el grupo ND. Este resultado se ha replicado de manera consistente en otros estudios en los que se han utilizado diferentes manipulaciones de la probabilidad de reforzamiento (e.g., Kruse \& Overmier, 1982; Santi \& Roberts, 1985; Urcuioli, 1990).

En varios trabajos se ha documentado que una variable adicional al intervalo de retención (i.e., intervalo EM-ECO) que también afecta a los índices de precisión o discriminación es la demora de reforzamiento (e.g., D'Amato \& Cox, 1976; McCarthy \& Davison, 1986, 1991; Sargisson \& 
White, 2003; Weavers, Foster \& Temple, 1998; Wilkie \& Spetch, 1978). Por ejemplo, McCarthy y Davison (1986) utilizando una tarea de igualación a la muestra, reportaron los efectos de variar el intervalo de retención y la demora de reforzamiento sobre la precisión de la discriminación. Reportaron que ambas manipulaciones resultaron en un decremento en la precisión del responder.

Recientemente, Sargisson y White (2003) evaluaron los efectos de variar la demora de reforzamiento y la demora entre estímulos (intervalo de retención) sobre la precisión del responder. Variaron entre sesiones la duración del intervalo de retención con valores que oscilaron desde 0 hasta $8 \mathrm{~s}$; mientras que entre fases, variaron la duración de la demora de reforzamiento usando los mismos valores que en el caso del intervalo de retención. Reportaron decrementos en la precisión del responder tanto por alargar la duración del intervalo de retención como por incrementar la demora de reforzamiento.

El presente estudio se diseñó con el propósito de evaluar la contribución que tiene el entrenamiento con consecuencias diferenciales, sobre la precisión del responder cuando se introducen dos tipos de variables disruptoras de la ejecución (intervalo de retención y demora de reforzamiento). Adicionalmente, el trabajo extendió los hallazgos reportados por DeLong y Wasserman (1981) hacia una tarea de discriminación condicional con ratas, empleando diferentes probabilidades de reforzamiento como una variación cuantitativa de las consecuencias diferenciales. Específicamente, se entrenaron a ratas en una tarea de discriminación condicional; para un grupo (Grupo CD) se reforzaron las respuestas correctas con diferentes probabilidades de reforzamiento (0.8 vs. 0.4) dependiendo de la señal condicional con la que iniciara el ensayo; mientras que para otro grupo de ratas (Grupo CND), las respuestas correctas fueron reforzadas con la misma probabilidad (0.6), independientemente de la señal condicional; posteriormente, se introdujeron dos tipos de demoras, demora entre la señal condicional y la fase de elección (presentación de las opciones de respuesta) y demora de reforzamiento.

\section{Método}

\section{Sujetos}

Se usaron 6 ratas macho Wistar de tres meses de edad e ingenuas experimentalmente, con alimentación libre y bajo un régimen de privación de agua por $23.5 \mathrm{~h}$. Cada sujeto recibió libre acceso al agua durante 30 minutos posteriores a la finalización de cada sesión experimental.

\section{Aparatos}

Se utilizaron 3 cámaras experimentales para ratas marca MED (ENV-008), cada caja estuvo equipada con un dispensador de agua (ENV-202M) y dos palancas. Las palancas se encontraban a $2.5 \mathrm{~cm}$ del piso y requirieron de una fuerza de $0.15 \mathrm{~N}$ para cerrar el microswitch. Como reforzador se utilizó una gota de agua de $0.01 \mathrm{cc}$ que se presentó por medio de la activación del dispensador de agua. Cada cámara experimental se colocó dentro de un cubículo de aislamiento acústico (ENV-022M) con un ventilador que sirvió como ruido blanco y facilitó la circulación del aire al interior de la cámara. La programación, registro y colección de eventos se realizaron mediante un equipo de cómputo, una interfase y el software MED-PC IV para ambiente Windows.

\section{Procedimiento}

Pre-entrenamiento: Cada ensayo iniciaba con el encendido de la luz general y de un reloj que proporcionaba una gota de agua cada $60 \mathrm{~s}$ sin requerimiento de respuesta (TF 60 s). Complementariamente, se presentaba una de las dos palancas de manera aleatoria en cada uno de los ensayos. Las respuestas a la palanca retraían la misma y activaban el bebedero por $3 \mathrm{~s}$. Posterior a la entrega de la gota de agua, iniciaba un intervalo entre ensayos (IEE) de 60 s. Los sujetos estuvieron en estas condiciones durante 4 sesiones de 60 ensayos cada una.

Entrenamiento (adquisición): Cada ensayo comenzaba con el encendido de la luz general y la activación de un tono (fijo o intermitente) con una 
duración de $5 \mathrm{~s}$, inmediatamente después del tono, se insertaban las dos palancas dentro de la cámara experimental. Las palancas no se retraían hasta que se emitía una respuesta en cualquiera de ellas. Para todos los sujetos, se consideraron ensayos correctos aquellos cuya respuesta a la palanca izquierda era precedida por el tono fijo, y aquellos en los que la respuesta a la palanca derecha era posterior a la presentación del tono intermitente. Para los sujetos del grupo con consecuencias diferenciales (CD), las respuestas a la palanca izquierda posteriores al tono fijo tuvieron una de probabilidad de reforzamiento de 0.8 ; mientras que las respuestas a la palanca derecha seguida del tono intermitente tuvieron una probabilidad de reforzamiento de 0.4. Las condiciones fueron idénticas para el grupo de consecuencias no diferenciales (CND), excepto que las respuestas correctas a cualquiera de las palancas tuvieron una probabilidad de reforzamiento de 0.6 independientemente de la señal condicional con la que iniciara el ensayo (tono fijo o tono intermitente). Las respuestas correctas activaban el bebedero y simultáneamente apagaba la luz general. Cuando la probabilidad no determinaba la entrega del agua, solamente se suspendía la luz general y daba inicio un IEE de 45 s. Las respuestas incorrectas no tuvieron ninguna consecuencia programada, únicamente que daba inicio del IEE. Los sujetos se mantuvieron en estas condiciones durante 26 sesiones de 60 ensayos cada una (línea base 1).

Fase de Demora (intervalo de retención): Al terminar la fase de adquisición, los dos grupos de sujetos fueron expuestos a 4 sesiones adicionales bajo las mismas condiciones que en la fase anterior, excepto por la introducción de una demora de $4 \mathrm{~s}$ (intervalo de retención) que iniciaba entre la finalización del tono fijo o intermitente y la inserción de las palancas en la cámara experimental. Para guardar cierto grado de comparabilidad con otros trabajos (e.g., Sargisson \& White, 2003), el valor de demora se determinó como la media de los valores de demora reportados por Williams (1998, Exp. 1 y 2).

Re-establecimiento: Concluidas las cuatro sesiones de la fase anterior, los sujetos fueron colocados nuevamente en las mismas condiciones a las de la fase de adquisición durante 4 sesiones adicionales (línea base 2).

Fase de Demora (Demora de reforzamiento): Por último, durante cuatro sesiones los sujetos fueron colocados en una tarea similar a la de la fase de demora 1, excepto que ahora la demora fue entre la respuesta y la entrega de la gota de agua (demora de reforzamiento). Después de la respuesta correcta, se demoró la entrega del reforzador durante $4 \mathrm{~s}$. La duración de la demora de reforzamiento siguió el mismo criterio que el empleado en el caso del intervalo de retención, permitiendo hacer comparaciones directas de los efectos de ambas demoras con la misma duración, como se ha reportado en otros trabajos (e.g., Sargisson \& White, 2003).

\section{Resultados}

En la Figura 1, se presenta el índice de discriminación en bloques de dos sesiones, promedio de los sujetos del grupo entrenado con consecuencias diferenciales $(\mathrm{CD})$ y de los sujetos del grupo entrenado con consecuencias no diferenciales (CND). En esta figura, se observa que durante las primeras sesiones el índice de discriminación es ligeramente superior en el Grupo CND; sin embargo, con el transcurso de las sesiones de entrenamiento se encontró que el Grupo CD mostró una velocidad de adquisición mucho más rápida que la observada en el Grupo CND. También se observa que los niveles terminales de discriminación son superiores en el Grupo CD. El índice de discriminación terminal, promedio de los últimos dos bloques para el Grupo CD fue de 0.88 , mientras que para el Grupo CND fue de 0.74 .

La introducción de una demora entre la SC y la oportunidad para responder resultaron en un decremento en los índices de discriminación en ambos grupos. En el grupo CD, el índice promedio tuvo un decremento a 0.79 , mientras que en el grupo CND, el índice se redujo hasta 0.58. Un análisis de varianza para medidas repetidas (ANOVA) entre sujetos (grupo) y uno intrasujetos (bloques de sesiones) mostraron un efecto principal entre grupos (CD vs. CND), $F(1,4)=16.413, p<0.01$ y efectos principales con el transcurso de los bloques 
de sesiones, $F(1,12)=57.074, p<0.01$, también se encontró una interacción significativa entre los bloques de sesiones y el grupo (sesiones $\mathrm{X}$ grupo), $F(12,48)=16.399, p<0.01 ;$ confirmando que los sujetos del grupo CD mostraron índices de discriminación superiores y una mayor velocidad de adquisición que los sujetos del grupo CND.

Durante el reestablecimiento (línea base 2) se observó que los índices de discriminación incrementaron en ambos grupos, para el grupo CD el índice promedio incrementó hasta 0.83 , mientras que para el grupo CND fue de 0.78. La introducción de la demora de reforzamiento resultó en decrementos en los índices de discriminación en ambos grupos; sin embargo, a diferencia de los efectos observados con la introducción del intervalo de retención, en este caso no se observaron diferencias en los índices de discriminación entre ambos grupos, CD (0.72) y NCD (0.73).

Una manera de estimar la diferencia entre los niveles terminales de discriminación en la fase de adquisición y en la fase en la que se introdujo la demora, es por medio de la proporción de cambio. Esta medida ha sido útil para representar los cambios observados como resultado de la introducción de variables disruptoras (e.g., demora entre estímulos, demora de reforzamiento) en el área de resistencia al cambio (e.g., Nevin \& Grace, 2000; Nevin, Milo, Odum \& Shahan, 2003; Odum, Shahan \& Nevin, 2005; Shahan \& Lattal, 2005). Es por ello que, en la Figura 2, se presenta la proporción de cambio en el índice de discriminación en cada fase de demora relativo al índice promedio en los últimos dos bloques de cada línea base precedente (adquisición y línea base 2). En esta figura se puede observar que en el Grupo CND se presentó un mayor decremento (i.e., menor resistencia al cambio) en el índice de discriminación como resultado de incrementar el intervalo de retención. La proporción de cambio en el Grupo CND fue de 0.76, mientras que en el Grupo CD fue 0.93. Una prueba $t$ para grupos independientes confirmó diferencias significativas entre grupos $t(4)=3.88$, $p=0.017$. La introducción de la demora de refor-

\section{FIGURA 1}

Índice de discriminación promedio de los sujetos en cada grupo. Los círculos negros representan los datos del grupo con consecuencias diferenciales (CD), los círculos blancos corresponden al grupo con consecuencias no diferenciales (CND). Las líneas verticales sobre cada punto corresponden al error estándar.

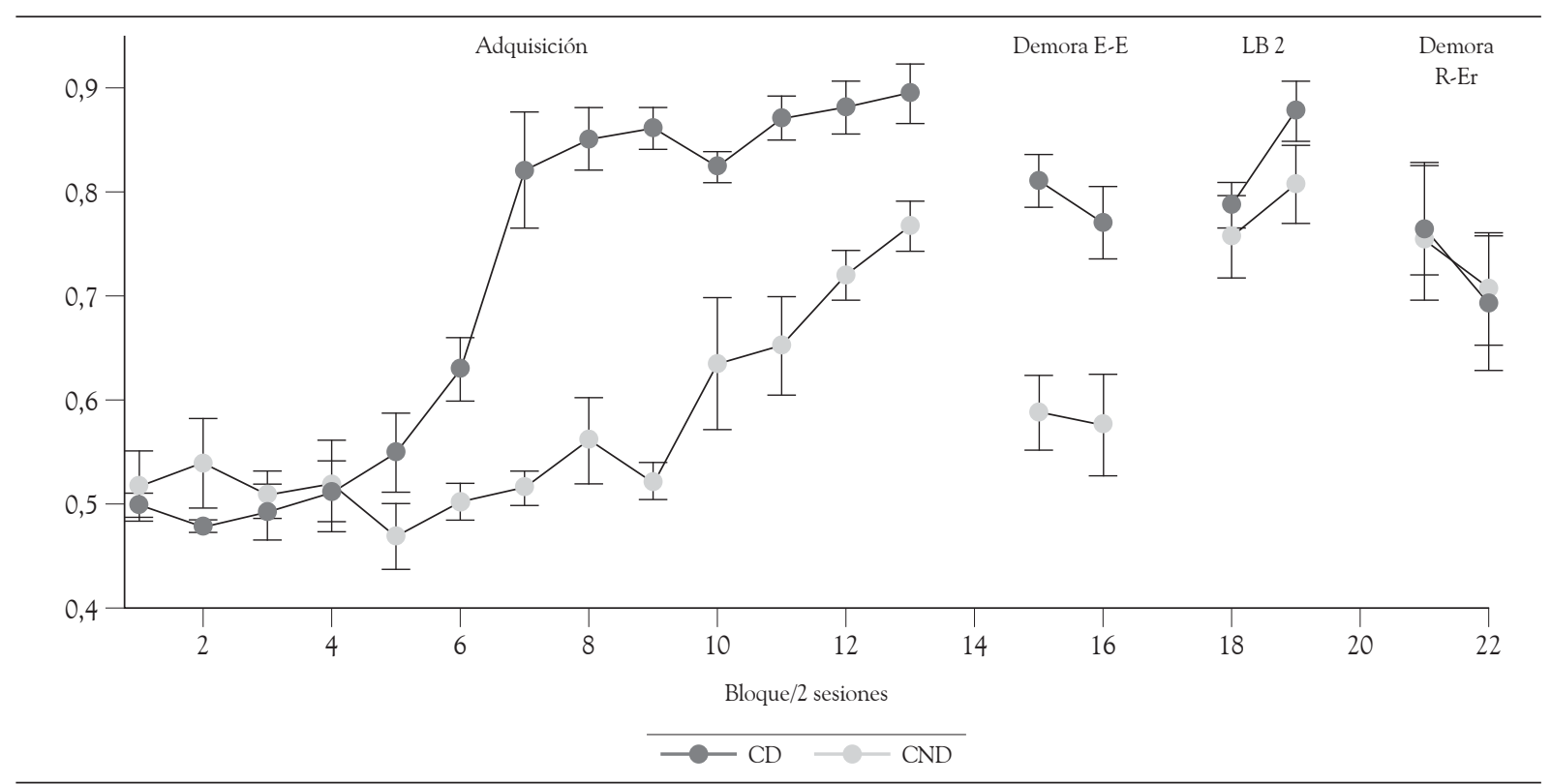

Fuente: elaboración propia. 
zamiento no mostró diferencias significativas en la proporción de cambio entre el grupo CD y CND (0.91 y 0.93 , respectivamente), una prueba $t$ para muestras independientes confirmó la ausencia de diferencias entre grupos $t(4)=-0.66, p=0.545$.

\section{Figura 2}

Proporción de cambio del índice de discriminación promedio de los sujetos de cada grupo en cada fase de demora. En cada barra se muestra el error estándar con la línea vertical.

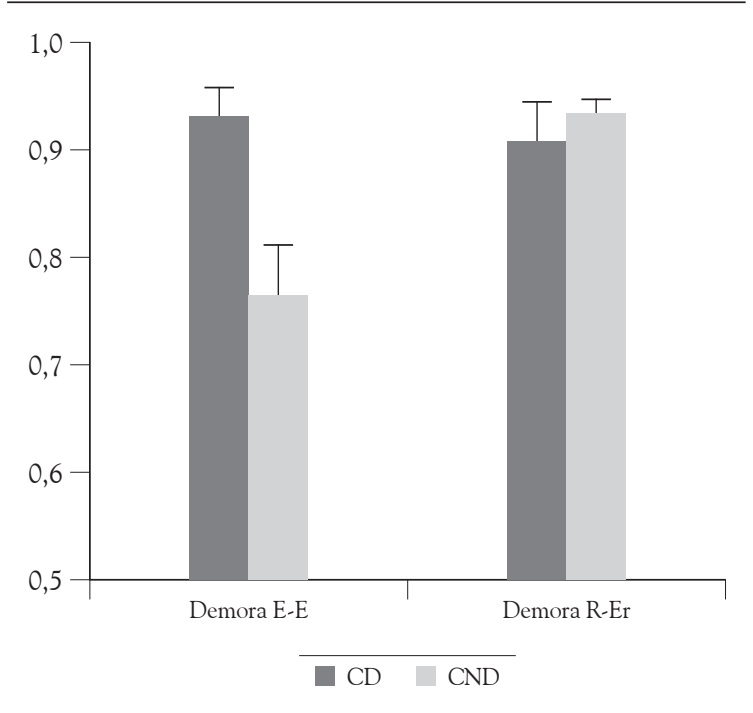

Fuente: elaboración propia.

\section{Discusión}

El propósito del presente estudio fue evaluar la contribución que tiene el procedimiento de consecuencias diferenciales, impuesto mediante la probabilidad diferencial de reforzamiento, sobre el aprendizaje de discriminaciones condicionales, y su efecto sobre la precisión de la discriminación en condiciones de demora entre estímulos (i.e. intervalo de retención) y demora de reforzamiento.

De manera general, se encontró que los sujetos entrenados en un procedimiento con consecuencias diferenciales (CD), mostraron una mayor velocidad de adquisición y más elevados niveles terminales de precisión que los sujetos entrenados en un procedimiento con consecuencias no diferenciales $(\mathrm{CND})$. El intervalo de retención resultó en un mayor decremento en los índices de discriminación, en los sujetos en los que las respuestas correctas fueron reforzadas con la misma probabilidad independientemente de la señal condicional (Grupo CND). Estos resultados son consistentes con los estudios que han reportado el ECD (e.g., Urcuioli, 2005) y extienden su generalidad a una tarea de discriminación condicional de dos opciones (i.e., two-choice) con ratas en la que se varía la probabilidad de reforzamiento (e.g., DeLong \& Wasserman, 1981; Kruse \& Overmier, 1982).

La imposición de una demora de reforzamiento no modificó diferencialmente la precisión del responder, en ninguno de los grupos. Los resultados mostrados en la Figura 2 dejan ver claramente que el efecto del intervalo de retención se ve aminorado por el uso de entrenamientos con consecuencias diferenciales; mientras que los efectos de la demora de reforzamiento prácticamente no modifican el índice de discriminación.

Varios trabajos han reportado que alargar la duración de la demora de reforzamiento resulta en decrementos moderados de la precisión, mientras que alargar la duración del intervalo de retención resulta en marcados y progresivos decrementos (e.g., McCarthy \& Davison, 1986; Sargisson \& White, 2003).

Por ejemplo, Sargisson y White (2003) evaluaron los efectos conjuntos de variar tanto la duración de la demora de reforzamiento como de la duración del intervalo de retención. La manipulación del intervalo de retención se realizó entre sesiones con valores de 0, 2, 4, 6 y 8 s; mientras que la demora de reforzamiento se varió entre fases utilizando las mismas duraciones empleadas, para evaluar los efectos de intervalo de retención. Los autores reportaron que la precisión decrementó tanto por alargar la duración del intervalo de retención como por incrementar la demora de reforzamiento; sin embargo, el efecto de alargar la demora de reforzamiento resultó en menores decrementos que los observados por alargar la duración del intervalo de retención.

En otro trabajo, McCarthy y Davison (1986), utilizando una tarea de igualación a la muestra, reportaron los efectos de variar la duración del 
intervalo de retención y la demora de reforzamiento; de manera consistente con los resultados reportado por Sargisson y White (2003), encontraron que ambas manipulaciones resultaron en un decremento en la precisión del responder, y que nuevamente el decremento en la precisión al incrementar la demora de reforzamiento fue menor que el decremento en la precisión, como resultado de alargar la duración del intervalo de retención.

Davison y Nevin (1999) ofrecieron un modelo para explicar las ejecuciones en tareas de discriminación condicional asumiendo que la precisión del responder está determinada tanto por la discriminabilidad entre los estímulos (intervalo de retención) como por la discriminabilidad entre las contingencias de reforzamiento (demora de reforzamiento), haciendo predicciones de diferentes ejecuciones que resultan de la interacción entre los efectos de la demora de reforzamiento y del intervalo de retención. En este modelo, la reducción en la discriminabilidad por incrementar la demora de reforzamiento es atribuida a un decremento o degradación de la contingencia respuesta-reforzador. En este sentido, los resultados del presente estudio muestran un decremento o degradación de la discriminabilidad tanto en el Grupo CD como en el Grupo CND, cuando se introdujo la demora de reforzamiento.

Sin embargo, la ausencia de diferencias en el índice de discriminación entre ambos grupos no puede ser interpretada a la luz de la propuesta formulada por Davison y Nevin. Es posible que el decremento en el porcentaje de respuestas correctas y que el mantenimiento en la precisión ante la demora de reforzamiento, puedan deberse a que los intervalos temporales estímulo-estímulo (i.e., intervalo de retención) y respuesta-reforzador (i.e., demora de reforzamiento) jueguen papeles diferentes en el control de la precisión (e.g., Davison \& Jenkins, 1985; Williams, 1998, 2003).

Davison y Jenkins (1985) mencionaron que alargar el intervalo de retención resulta en una pérdida gradual del control que tiene la señal al momento ante el cual el sujeto responde; mientras que cuando se incrementa la demora de reforzamiento, el control por el reforzador subsecuente a la respuesta de elección se reduce, de este modo los incrementos en el intervalo respuesta-reforzador son entendidos como una degradación del control que tiene el reforzador de la respuesta de la que es contingente el evento de estímulo.

La ausencia de cambios en la precisión por alargar la demora de reforzamiento, también fue recientemente reportada por Flores y Mateos (2009, Experimento 2) quienes compararon las ejecuciones de sujetos entrenados en una tarea de discriminación condicional con diferentes duraciones del intervalo entre ensayos.

Flores y Mateos entrenaron a dos grupos de ratas en un procedimiento de discriminación condicional con las mismas características a las empleadas en el presente estudio. Para un grupo de ratas el intervalo entre ensayos (IEE) se mantuvo constante en $45 \mathrm{~s}$, mientras que para el otro grupo la duración del IEE se incrementó a medida que también incrementaba la duración de la demora de reforzamiento, en ambos grupos las demoras de reforzamiento incrementaron entre fases con valores de 0, 2, 4 y 8 s. Flores y Mateos reportaron que en ambos grupos el porcentaje de respuestas correctas (i.e., índice de discriminación) se mantuvo sin cambios ante los diferentes valores de demora de reforzamiento.

Siguiendo a Williams (1998), Flores y Mateos explicaron sus resultados considerando que los efectos de las variables disruptoras de la ejecución (i.e., intervalo de retención y demora de reforzamiento) tienen propiedades funcionalmente diferentes y juegan papeles distintos en el aprendizaje de tareas que implican contingencias estímuloestímulo y respuesta-reforzador.

La ausencia de efectos por alargar la demora de reforzamiento sigue siendo una interrogante que reclama investigación sistemática adicional, que permita ofrecer una interpretación clara de este efecto.

\section{Referencias}

Alling, K., Nickel, M. \& Poling, A. (1991). The effects of differential and nondifferential outcomes on response rates and accuracy under delayed mat- 
ching to sample procedure. The Psychological Record, 41, 537-549.

Brodigan, D. \& Peterson, G. (1976). Two-choice conditional discrimination performance of pigeon as a function of reward expectancy, prochoice delay, and domesticity. Animal Learning and Behavior, 4(2), 121-124.

Carlson, J. \& Wielkiewicz, R. (1972). Delay of reinforcement in instrumental discrimination learning of rats. Journal of Comparative and Physiological Psychology, 81 (2), 365-370.

Chatlosh, D. \& Wasserman, E. (1992). Memory and expectancy in delayed discrimination procedures. En I. Gormezano \& E. A. Wasserman (Eds.), Learning and Memory (pp. 61-79). New Jersey: Lawrence Erlbaum Associates.

Cumming, W. W. \& Berryman, R. (1961). Some data on matching behavior in the pigeon. Journal of the Experimental Analysis of Behavior, 4, 281-284.

Cumming, W. W. \& Berryman, R. (1965). The complex discriminated operant: Studies of matching to sample and related problems. En D. I. Mostofsky (Ed.), Stimulus Generalization (pp. 284-330). Stanford: Stanford University Press.

D’Amato, M. R. \& Cox, J. K. (1976). Delay of consequences and short-term memory in monkeys. En D. L. Medin, W. A. Roberts \& R. T. Davis (Eds.), Processes of animal memory (pp. 49-78). Mahwah, NJ: Erlbaum.

Davison, M. \& Jenkins, P. E. (1985). Stimulus discriminability, contingency discriminability, and schedule performance. Animal Learning and Behavior, 13, 77-84.

Davison, M. \& Nevin, J. (1999). Stimuli, reinforcers, and behavior: An integration. Journal of the Experimental Analysis of Behavior, 71, 439-482.

DeLong, R. E. \& Wasserman, E. A. (1981). Effects of differential reinforcement expectancies on successive matching-to-sample performance in pigeons. Journal of Experimental Psychology: Animal Behavior Processes, 7, 394-412.

Flores, C., Arriaga, P. \& Ortiz, R. (2006). Contribución de intervalos entre ensayos diferenciales en tareas de igualación a la muestra demorada. Universitas Psychologica, 5(1), 139-146.
Flores, C. \& Mateos, R. (2009). Tiempo relativo, elección demorada y demora de reforzamiento en discriminación condicional. Revista Mexicana de Análisis de la Conducta, 35, 135-149.

Goeters, S., Blakely, E. \& Poling, A. (1992). The differential outcome effect. The Psychological Record, 42, 389-411.

Kruse, J. M. \& Overmier, J. B. (1982). Anticipation of reward omission as a cue for choice behavior. Learning and Motivation, 13, 505-525.

McCarthy, D. C. \& Davison, M. (1986). Delayed reinforcement and delayed choice in symbolic matching to sample: Effects on stimulus discriminability. Journal of the Experimental Analysis of Behavior, 46, 293-303.

McCarthy, D. C. \& Davison, M. (1991). The interaction between stimulus and reinforcer control on remembering. Journal of the Experimental Analysis of Behavior, 56, 51-66.

Nevin, J. A. \& Grace, R. C (2000). Behavioral momentum and the law of effect. Behavioral and Brian Sciences, 23, 73-130.

Nevin, J. A., Milo, J., Odum, A. L. \& Shahan, T. A. (2003). Accuracy of discrimination, rate of responding, and resistance to change. Journal of the Experimental Analysis of Behavior, 73, 307-321.

Odum, A. L., Shahan, T. A. \& Nevin, J. A. (2005). Resistance to change of forgetting functions and response rates. Journal of the Experimental Analysis of Behavior, 84, 65-75.

Ortíz, R. \& Flores, C. (2007). Efectos de variar la posición temporal del estímulo intrusivo durante el intervalo de demora y de la consistencia e inconsistencia de la relación muestra-reforzador. Universitas Psychologica, 6, 451-463.

Overmier, B., Bull, J. \& Trapold, M. (1971). Discriminative cue properties of different fears and their role in response selection in dogs. Journal of Comparative and Physiological Psychology, 76(3), 478-482.

Overmier, J. B., Savage, L. M. \& Sweeney, W. A. (1999). Behavioral and pharmacological analyses of memory: New behavioral options for remediation. En M. Haug \& R. E. Whalen (Eds.), Animal models of human emotion and cognition (pp. 231-245). Washington, DC: American Psychological Association. 
Pérez, G. L. (2001). Procesos de aprendizaje de discriminaciones condicionales. Psicotehma, 13, 650-658.

Peterson, G., Wheeler, R. \& Armstrong, G. (1978). Expectancies as mediators in the differential reward conditional performance of pigeons. Animal Learning and Behavior, 6(3), 279-285.

Peterson, G., Wheeler, R., \& Trapold, M. (1980). Enhancement of pigeons' conditional discrimination performance of reinforcement and non reinforcement. Animal Learning and Behavior, 8, 1, 22-30.

Ramos, R. \& Savage, L. M. (2003). The differential outcomes procedure can interfere or enhance operant rule learning. Integrative Physiological \& Behavioral Science, 38, 17-35.

Santi, A. \& Roberts, W. A. (1985). Prospective representations: The effects of varied mapping of sample stimuli to comparison stimuli and differential trial outcomes on pigeons` working memory. Animal Learning E Behavior, 13, 103-108.

Sargisson, R. J. \& White, K. G. (2003). The effect of reinforcer delays on the form of the forgetting function. Journal of the Experimental Analysis of Behavior, 80, 77-94.

Shahan, T. \& Lattal, K. A. (2005). Unsignaled delay of reinforcement, relative time, and resistance to change. Journal of the Experimental Analysis of Behavior, 83, 201-219.
Urcuioli, P. J. (1990). Some relationships between outcome expectancies and sample stimuli in pigeons' delayed matching. Animal Learning \& Behavior, 18, 302-314.

Urcuioli, P. J. (2005). Behavioral and associative effects of differential outcomes in discrimination learning. Learning \& Behavior, 33, 1-21.

Weavers, R., Foster, T. M. \& Temple, W. (1998). Reinforcer efficacy in a delayed matching-to-sample task. Journal of the Experimental Analysis of Behavior, 69, 77-85.

Wilkie, D. M. \& Spetch, M. L. (1978). The effect of sample and comparison ratio sachedules on delayed matching to sample in the pigeon. Animal Learning \& Behavior, 6, 273-278.

Williams, B. A. (1998). Relative time and delay of reinforcement. Learning and motivation, 29, 236-248.

Williams, B. A. (2003). Challenges to timing-based theories of operant behavior. Behavioural Processes, 62, 115-123.

Williams, D., Butler, M. \& Overmier, B. (1990). Expectancies of reinforcer location and quality as cues for a conditional discrimination in pigeons. Journal of Experimental Psychology: Animal Behavior Processes, 16(1), 3-13. 
